\title{
Corrigendum
}

\section{Strategy-proof and individually rational social choice functions for public good economies}

\author{
Shigehiro Serizawa ${ }^{\star}$ \\ Department of Economics, Shiga University, 1-1-1, Banba, Hikone, Shiga 522, JAPAN \\ Received: July 15, 1996
}

Summary. This note is to inform about a mistake in my paper (Serizawa, 1996). In that paper, I characterized strategy-proof, individually rational, budget-balancing, non-exploitative and non-bossy social choice functions for economies with one public good and one private good. I established as Theorem 3 (page 507) that a social choice function is strategy-proof, individually rational with respect to endowment, budget-balancing, nonexploitative and non-bossy if and only if it is a scheme of "semi-convex cost sharing determined by the minimum demand principle". I also exposed one example (Example 2, page 507) in order to emphasize that non-bossiness is indispensable for this characterization. I claimed that the social choice function in that example satisfies the above axioms except for non-bossiness, and is not a scheme of semi-convex cost sharing. However, the social choice function in the example is actually not strategy-proof, as shown in the simple discussion below. Therefore it is an open question whether or not a similar characterization theorem holds without non-bossiness.

JEL Classification Numbers: C71, D82, H41.

In this note, I use the same model, assumptions and definitions as Serizawa (1996). Thus I omit them here. In the following, I expose Theorem 3 and Example 2 of Serizawa (1996), and then show that the social choice function in the example is not strategy-proof.

Theorem $3^{1}$. A social choice function is strategy-proof, e-individually rational, non-exploitative and non-bossy if and only if it is a scheme of semi-convex cost sharing determined by the minimum demand principle.

\footnotetext{
` I thank Professor Rajat Deb, who kindly pointed out my mistake.

${ }^{1}$ Budget-balance is assumed in the beginning of the paper (Serizawa, 1996). Thus it is not mentioned in the theorem.
} 
Example 2. There are three agents, say, agent 1,2 and 3. The cost function $C$ of the public good is linear, that is, $C(y)=y$ for any production level $y \in \mathbf{R}_{+}$ of the public good. The endowment $e$ is the allocation such that all the agents have one unit of the private good and no public good is produced. The allocation $a^{1}$ (respectively $a^{2}$ ) is the allocation such that one unit of the public good is produced, agent 1 (respectively agent 2) pays the whole cost, and the other agents consume their endowment of the private good. The social choice function $f$ is specified as follows. Let $u=\left(u^{1}, u^{2}, u^{3}\right)$ be a preference profile, where $u^{i}, i=1,2,3$, denote agent $i^{\prime}$ 's preference respectively. If $u^{1}(e) \geq u^{1}\left(a^{1}\right)$ or $u^{2}(e) \geq u^{2}\left(a^{2}\right)$, then $f(u)=e$; and if $u^{1}\left(a^{1}\right)>u^{1}(e), u^{2}\left(a^{2}\right)>u^{2}(e)$ and the slope of agent 3 's indifference curve through $(1,1)$ is "steep" (respectively "not steep"), then $f(u)=a^{1}$ (respectively $f(u)=a^{2}$ ).

Now I show that the social choice function $f$ in the example is not strategy-proof. Let $u=\left(u^{1}, u^{2}, u^{3}\right)$ be the preference profile such that $u^{1}\left(a^{1}\right)>u^{1}(e), u^{2}\left(a^{2}\right)>u^{2}(e)$ and the slope of agent 3 's indifference curve through $(1,1)$ is steep. Then $f(u)=a^{1}$. Let $\hat{u}^{2}$ be agent 2's preference such that $\hat{u}^{2}\left(a^{2}\right)<\hat{u}^{2}(e)$. Then since $f\left(u^{1}, \hat{u}^{2}, u^{3}\right)=e$ and $\hat{u}^{2}\left(a^{1}\right)>\hat{u}^{2}(e)$, it follows that $\hat{u}^{2}(f(u))>\hat{u}^{2}\left(f\left(u^{1}, \hat{u}^{2}, u^{3}\right)\right)$. Hence $f$ is not strategy-proof.

I apologize to the readers for the inconvenience owing to this mistake.

\section{Reference}

Serizawa, S: Strategy-proof and individually rational social choice functions for public good economies. Economic Theory 7, 501-512 (1996) 